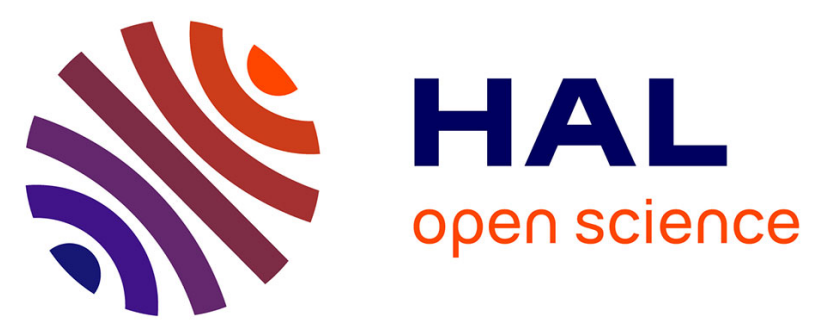

\title{
Inhibition of HIV-1 by Non-Nucleoside Reverse Transcriptase Inhibitors via an Induced Fit Mechanism - Importance of Slow Dissociation and Relaxation Rates for Antiviral Efficacy
}

Malin Elinder, Philippe Selhorst, Guido Vanham, Bo Öberg, Lotta Vrang, U. Helena Danielson

\section{To cite this version:}

Malin Elinder, Philippe Selhorst, Guido Vanham, Bo Öberg, Lotta Vrang, et al.. Inhibition of HIV-1 by Non-Nucleoside Reverse Transcriptase Inhibitors via an Induced Fit Mechanism - Importance of Slow Dissociation and Relaxation Rates for Antiviral Efficacy. Biochemical Pharmacology, 2010, 80 (8), pp.1133. 10.1016/j.bcp.2010.06.035 . hal-00618192

\author{
HAL Id: hal-00618192 \\ https://hal.science/hal-00618192
}

Submitted on 1 Sep 2011

HAL is a multi-disciplinary open access archive for the deposit and dissemination of scientific research documents, whether they are published or not. The documents may come from teaching and research institutions in France or abroad, or from public or private research centers.
L'archive ouverte pluridisciplinaire HAL, est destinée au dépôt et à la diffusion de documents scientifiques de niveau recherche, publiés ou non, émanant des établissements d'enseignement et de recherche français ou étrangers, des laboratoires publics ou privés. 


\section{Accepted Manuscript}

Title: Inhibition of HIV-1 by Non-Nucleoside Reverse Transcriptase Inhibitors via an Induced Fit Mechanism Importance of Slow Dissociation and Relaxation Rates for Antiviral Efficacy

Authors: Malin Elinder, Philippe Selhorst, Guido Vanham, Bo

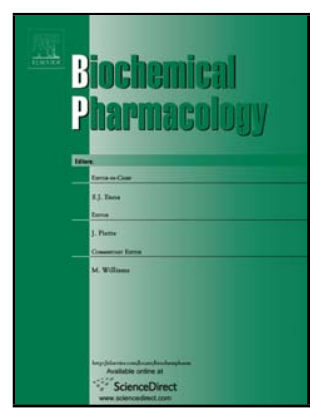

Öberg, Lotta Vrang, U. Helena Danielson

PII:

S0006-2952(10)00469-7

DOI: doi:10.1016/j.bcp.2010.06.035

Reference: BCP 10616

To appear in: $\quad B C P$

Received date: $\quad 30-4-2010$

Revised date: $\quad 18-6-2010$

Accepted date: $\quad$ 21-6-2010

Please cite this article as: Elinder M, Selhorst P, Vanham G, Öberg B, Vrang L, Danielson UH, Inhibition of HIV-1 by Non-Nucleoside Reverse Transcriptase Inhibitors via an Induced Fit Mechanism - Importance of Slow Dissociation and Relaxation Rates for Antiviral Efficacy, Biochemical Pharmacology (2010), doi:10.1016/j.bcp.2010.06.035

This is a PDF file of an unedited manuscript that has been accepted for publication. As a service to our customers we are providing this early version of the manuscript. The manuscript will undergo copyediting, typesetting, and review of the resulting proof before it is published in its final form. Please note that during the production process errors may be discovered which could affect the content, and all legal disclaimers that apply to the journal pertain. 


\title{
Inhibition of HIV-1 by Non-Nucleoside Reverse Transcriptase
}

\section{Inhibitors via an Induced Fit Mechanism - Importance of Slow} Dissociation and Relaxation Rates for Antiviral Efficacy

Malin Elinder, ${ }^{\mathrm{a}}$ Philippe Selhorst, ${ }^{\mathrm{b}}$ Guido Vanham, ${ }^{\mathrm{b}}$ Bo Öberg, ${ }^{\mathrm{c}}$ Lotta Vrang, ${ }^{\mathrm{c}}$ and U. Helena Danielson ${ }^{\mathrm{a}}$

${ }^{a}$ Department of Biochemistry and Organic Chemistry, Uppsala University, BMC, Box 576, SE -75123 Uppsala, Sweden. ${ }^{\mathrm{b}}$ Department of Microbiology, Institute of Tropical Medicine, Nationalestraat 155, B-2000 Antwerp; Department of Biomedical Sciences, University of Antwerp; Faculty of Medicine and Pharmacy, Free University of Brussels; Belgium. ${ }^{\mathrm{c}}$ Medivir AB, Box 1086, SE-141 22 Huddinge, Sweden.

*Corresponding author: Department of Biochemistry and Organic Chemistry, Uppsala University, Box 576, SE-751 23 Uppsala, Sweden. Phone: +46 1847145 45. Fax +46 1855 84 31. E-mail: helena.danielson@biorg.uu.se.

Malin Elinder, malin.elinder@biorg.uu.se

Philippe Selhorst, PSelhorst@itg.be

Guido Vanham, GVanham@itg.be

Bo Öberg, bo.oberg@medivir.se

Lotta Vrang, lotta.vrang@medivir.se

Running title: Inhibition of HIV-1 by NNRTIs

\begin{abstract}
Abbreviations: AIDS, acquired immunodeficiency syndrome; Ba-L, bronchoalveolar lavage; dapivirine TMC-120; DA, detection area; DMSO, dimethylsulfoxide; DLV,delavirdine; EDC, 1-ethyl-3-(3-dimethylamino-propyl)carbodiimide; EFV;efavirenz; HIV-1, human immunodeficiency virus type 1; MOI, multiplicity of infection; IL-2, interleukin-2; NHS, Nhydroxysuccinimide; NNRTI, non-nucleoside reverse transcriptase inhibitor; NVP, nevirapine; PBMC, peripheral blood mononuclear cell; PETT, phenylethylthioazolylthiourea; PHA, phytohaemagglutinin; RT, reverse transcriptase; RU, resonance unit; SPR, surface plasmon resonance.
\end{abstract}




\section{ABSTRACT}

The importance of slow dissociation of non-nucleoside reverse transcriptase inhibitors (NNRTI) for antiviral effect has been investigated. The interaction kinetic characteristics of a series of NNRTIs and wild type and drug resistant variants of HIV-1 RT (EC 2.7.7.49) were analyzed by SPR biosensor technology. The antiviral effect was determined in MT-4 and peripheral blood mononuclear cells. Due to extremely slow dissociation rates and a complex interaction mechanism, rate constants could not be quantified. Instead, interaction characteristics were qualitatively analyzed using simulated sensorgrams. The simplest model describing these interactions adequately was an induced-fit mechanism, i.e. a mechanism involving the formation of an initial enzyme-inhibitor complex subsequently transformed into a more stable complex. Differences in rates of dissociation from the initial complex and rates of relaxation from the induced complex explained 1) the differences in the amounts of formed complex, 2) the stability of the complex and 3) the antiviral efficacies of the compounds. The effect of NNRTI binding site mutations also correlated with these kinetic characteristics. MIV-170 was the most effective inhibitor of wild type and mutant HIV-1 in cell culture, a property that was associated with the formation of the largest amount of complex and having the slowest relaxation and dissociation rates. This study supports the hypothesis that the efficacy of anti-HIV drugs requires slow dissociation from the target, thereby maximizing the duration of the inhibitory effect. It also illustrates the strength of simulating interaction data for qualitative analysis of tight-binding drugs and the importance of resolving the kinetic mechanism of drug-target interactions.

Keywords (max 6): HIV reverse transcriptase, NNRTI, MIV, kinetics, biosensor, SPR

\section{INTRODUCTION}

The continued spread of HIV is a major threat to individuals and societies in many parts of the world and problems with resistance development due to the therapeutic use of current 
non-nucleoside reverse transcriptase inhibitors (NNRTIs) are also increasing. All attempts to prevent transmission by the use of microbicides and vaccines have failed to date [1,2]. Many clinically evaluated microbicides lack preventive effect, which is not too surprising considering their non-specific mode of action, low potency, high protein binding and unselective irritating properties.

In the design of vaginal microbicides acting on a specific target, NNRTIs are the most promising compounds as active anti-HIV ingredients for a number of reasons [3]. These compounds are at least 1000 fold more efficient in inactivating virus than acid polymers, and much more efficient than nucleoside or nucleotide analogues. In addition, some NNRTIs may have the advantage of inhibiting both free virions and preventing infection of target cells by binding tightly to the reverse transcriptase [4]. Nevertheless, the usefulness of an NNRTI as a microbicide is expected to be dependent on its ability to rapidly and completely inactivate both wild type and mutant virus at a low concentration. MIV- 170 (Fig. 1) is a novel high affinity NNRTI that we have hypothesized to possess such characteristics and that consequently may have the potential to be used in a topical microbicide for prevention of HIV-1 transmission. A rapid metabolism of MIV-170 in human hepatocytes makes the compound less suitable for oral use (unpublished observations).

To establish the characteristics of MIV-170 with respect to its interaction with its target, we have analyzed the interaction kinetics of the compound with wild type and mutant HIV-1 RT using a surface plasmon resonance (SPR) biosensor. We have previously used this technology to characterize the interaction mechanism for NNRTIs with their target and to determine the resistance profiles of NNRTIs [5-7]. Due to the tight-binding interaction of the clinically used NNRTIs, the initial characterization was performed with a drug resistant variant from which the compounds dissociated. In subsequent studies, we have also used a biosensor-based approach to identify novel scaffolds and leads for design of anti-HIV drugs 
or microbicides by screening both fragment and focused libraries for compounds with suitable kinetic characteristics and resistance profiles $[8,9]$.

The present study was specifically designed to investigate how MIV-170 compared to the clinically used NNRTIs efavirenz, nevirapine, and delavirdine, as well as TMC-120 (dapivirine), an NNRTI with in vitro anti-HIV properties superior to those of the compounds used in the clinic today [10]. Since efficacy against drug resistant virus is essential, the study involved analysis of the interaction with wild type RT and K103N, Y181C, L100I mutants, as well as of the inhibition of replication of the corresponding viral strains in cell culture. In this study we were particularly interested in identifying the interaction kinetic parameters that correlate with a long lasting antiviral effect, hypothesized to be primarily an effect of slow dissociation. We therefore also determined the effect of the different compounds on the inhibition of viral replication in cell culture, using both MT4 and peripheral blood mononuclear cells (PBMCs).

\section{MATERIALS AND METHODS}

\subsection{Enzymes and inhibitors}

The wild type and three drug resistant enzyme variants (Y181C, K103N and L100I) of reverse transcriptase (EC 2.7.7.49) of HIV-1 (BH10 isolate) were obtained as previously [5, 11] described. All enzymes contained the additional substitution E478Q to abolish the RNase $\mathrm{H}$ activity. The purification procedure was simplified and only included three of the separation steps: anion exchange chromatography (Q Sepharose), affinity chromatography (Heparin Sepharose) and size exclusion chromatography (Superdex $\left.{ }^{\circledR}\right)$. All chromatography media was from GE Healthcare Bio-Sciences, Uppsala, Sweden. The enzymes were diluted in $5 \mathrm{mM}$ Hepes buffer ( $\mathrm{pH} 7.6,4 \mathrm{mM} \mathrm{MgCl}$ ) to a final concentration of $5 \mu \mathrm{g} / \mu \mathrm{L}$. 
The NNRTIs (MIV-170, delavirdine, efavirenz, nevirapine and TMC-120) were synthesized according to published procedures and references cited therein [12]. They were dissolved in $100 \%$ DMSO (Riedl-de-Haën, Seelze, Germany) and prepared as $20 \mathrm{mM}$ stock solutions in 96-well plates. For the viral replication assay in PBMC cultures, efavirenz, delavirdine and nevirapine were obtained through the NIH AIDS Research and Reference Reagent Program (Germantown, USA). TMC-120 was kindly provided by Tibotec BVBA, Mechelen, Belgium. Stock solutions of 10 to $50 \mathrm{mM}$ were made in 100\% DMSO. All compounds were used at non-toxic concentrations as determined by WST-1-tetrazolium based assays (data not shown).

\subsection{Biosensor experiments}

The interaction analyses were performed with a BIACORE ${ }^{\mathrm{TM}}$ A100 instrument (GE Healthcare Bio-Sciences, Uppsala, Sweden) at $25^{\circ}$. Reagents, buffers and BIACORETM Series S Sensor Chip CM5, research grade, were also from GE Healthcare Bio-Sciences, Uppsala, Sweden.

\subsubsection{Immobilization and methodology}

The four enzyme variants were immobilized in parallel detection areas (DA) on the sensor chip surface with standard amine coupling chemistry [9]. The enzymes were immobilized in the same positions in all four flow cells of the instrument: the wild type in DA 1, Y181C in DA 2, L100I in DA 4 and $\mathrm{K} 103 \mathrm{~N}$ in DA 5, as illustrated previously [9]. DA 3 was used as a reference and was only activated and deactivated. The enzymes were injected at a concentration of $0.2 \mu \mathrm{g} / \mu \mathrm{L}$ with a contact time of $1.5 \mathrm{~min}$. PBS-P (10 mM phosphate buffer $\mathrm{pH} 7.4,2.7 \mathrm{mM} \mathrm{KCl}, 0.14 \mathrm{M} \mathrm{NaCl}$ and $0.05 \%$ surfactant P20) was used as running buffer.

Since the protein surface could not be regenerated after injection of the NNRTIs, each surface was only used once and each chip could consequently only be used for analysis of 
four compounds. Compounds were injected in parallel in the four different flow cells, each containing the same panel of immobilized enzymes. Five different NNRTIs were tested in duplicate and one of them was injected on all three chips (i.e one time extra as a control) in order to enable comparisons of the data from the different chips. Each NNRTI was injected at $5.12 \mu \mathrm{M}$ over the surface at a flow rate of $30 \mu \mathrm{L} / \mathrm{min}$. The injection time was 1 minute and the dissociation was monitored for up to 10 minutes, but only the first 3.5 min was used in the analysis. Running buffer including 3\% DMSO was used as negative control and injected once before and once after the sample.

\subsubsection{Processing of data}

The raw signal $v s$. time data (sensorgrams) from the screening was processed with the BIACORETM A100 Evaluation 1.0 software (GE Healthcare Bio-Sciences, Uppsala, Sweden). The signals are reported in resonance units (RU). Non-specific signals were removed by subtraction of signals from the reference area. The data was normalized by dividing the signal by the molecular weight of the sample and then multiplied by 100 in order to get a scale from 0 to 100 . Corrections for differences in DMSO concentrations between sample and running buffer (bulk refractive index calibration) were also performed. Since the five inhibitors were tested over different chip surfaces, the sensorgrams had also to be corrected for differences in immobilization levels. This was done using the BIAevaluation Software 4.1 (GE Healthcare Bio-Sciences, Uppsala, Sweden) by dividing the obtained signal by the immobilization level in RU, multiplied by a factor of 10000 in order to get a scale in the same order of magnitude as the original data. Finally, the signal for a negative control was also subtracted.

\subsubsection{Simulation of sensorgrams}

Sensorgrams were simulated according to the principles and equations used in the simulation module of BIAevaluation software 4.1 (GE Healthcare Bio-Sciences, Uppsala, Sweden). Here, the model was defined as in Figure 3 and the rate equations as: $d_{1} I=$ 
$\left(k_{1} * \mathrm{I} * \mathrm{E}_{1}+k_{4} * \mathrm{E}_{2} \mathrm{I}\right)-\left(k_{2} * \mathrm{E}_{1} \mathrm{I}+k_{3} * \mathrm{E}_{1} \mathrm{I}\right) ; \mathrm{dE}_{2} \mathrm{I}=\left(k_{3} * \mathrm{E}_{1} \mathrm{I}\right)-\left(k_{4} * \mathrm{E}_{2} \mathrm{I}\right)$ and the simulations were performed with MatLab (The MathWorks, Inc., Natick, MA, United States).

\subsection{Viral replication inhibition assays}

Inhibition of HIV replication was measured in PBMC (kindly provided by the Antwerp Blood Transfusion Centre, Belgium) as a representative in vivo target of HIV-1 and in MT-4 cells as previously described [9]. The virus strain for the MT-4 assay was HIV-1IIIb (R.C. Gallo) and resistant virus containing the L100I, K103N or Y181C amino acid substitutions were isolated by in vitro selection from HIV-1IIIb, replicating in the presence of increasing concentrations of NNRTIs.

For the PBMC analysis, a wild type HIV-1 subtype B strain (Ba-L) was used. The inhibition was determined by pre-incubating $50 \mu \mathrm{L}$ of the compound with $50 \mu \mathrm{L}$ of cell-free virus at $10^{-3} \mathrm{MOI}$ in a transparent $96-$ well plate at $37^{\circ} \mathrm{C}$ and $5 \% \mathrm{CO}_{2}$ for $30 \mathrm{~min}$. The compound had been prepared as a serial dilution in interleukin-2 (IL-2) medium (RPMI 1640 medium containing $15 \%$ heat-inactivated fetal bovine serum (FBS) and $50 \mathrm{mg} / \mathrm{mL}$ gentamicine (Lonza, Verviers, Belgium) supplemented with $1 \mathrm{ng} / \mathrm{ml}$ IL-2 (Gentaur, Brussel, Belgium), $2 \mu \mathrm{g} / \mathrm{mL}$ polybrene (Sigma-Aldrich, Bornem, Belgium) and $5 \mu \mathrm{g} / \mathrm{mL}$ hydrocortisone (Calbiochem, Leuven, Belgium), with delavirdine and nevirapine used at 50000-10000-1000-100-10-1 nM, and TMC-120, MIV-170 and efavirenz used at 100001000-100-10-1-0.1 nM. Seventy five thousand PHA / IL-2 stimulated PBMCs (cultured for 48 hours in RPMI-1640 containing 10\% FBS and $50 \mathrm{mg} / \mathrm{ml}$ gentamicin supplemented with 2 $\mu \mathrm{g} / \mathrm{ml}$ phytohemagglutinin (PHA) (Remel, Kent, United Kingdom) following activation for 24h in IL-2 medium) in $100 \mu \mathrm{L} \mathrm{IL-2}$ medium were then added to each well and incubated for 24 hours. Subsequently, excess virus was removed and the cells were resuspended in fresh IL2 medium with the same concentration of compound as used in the incubation. Medium and compound were refreshed once more after one week of culture. On day 14, the p24 antigen 
concentration in the supernatant of these cultures was measured using an in-house ELISA

[13]. The experiment contained six replicates and was performed three times using PBMCs from different donors.

The compound mediated-inhibition of viral replication (as the percentage of the value for the untreated control) was plotted against the compound concentration. Non-linear regression analysis was performed using GraphPad Prism 5.02 (GraphPad Software, Inc, La Jolla, USA). The results were expressed as $\mathrm{EC}_{50}$ values, defined as the concentration of drug at which there was $50 \%$ viral replication as compared to the drug-free control.

\section{RESULTS}

\subsection{Interaction analysis}

The immobilization levels for the enzyme variants varied between 17300-18500 RU for the wild type, 5400-14400 RU for Y181C, 12300-14900 RU for L100I and 17500-18900 RU for K103N. All tested NNRTIs formed stable complexes with the proteins and regeneration of the sensor surface could therefore not be achieved without damaging the enzyme. Hence, only one concentration of each inhibitor could be injected over each enzyme surface. Since data from a series of concentrations of each inhibitor is needed for a reliable determination of maximal binding levels and kinetic constants, an experimental design that reduced the consumption of chips but still provided an adequate data set for qualitative kinetic information to be extracted from single compound injections was devised. A total of 11 injections were required for two replicates of each compound with one of these serving as a reference sample in another experiment, thus consuming $3\left(2 \frac{3 / 4}{4}\right)$ chips. The total consumption of each enzyme variant was $115.5 \mu \mathrm{g}(10.5 \mu \mathrm{g}$ per detection area, i.e. $42 \mu \mathrm{g}$ per full chip.)

The sensorgrams in Fig. 2 show that all of the studied inhibitors except nevirapine formed stable complexes with the wild type enzyme. The sensorgrams differed qualitatively 
with respect to the signal level at the end of the injection (i.e. at $60 \mathrm{~s}$ ) and after dissociation (i.e. at $200 \mathrm{~s}$ ), as well as to the slope of the sensorgram during the dissociation phase, seen most easily at the end (e.g. 150-200 s, shaded area). The sensorgrams for the two replicates for each compound were very similar. For most of the inhibitors, the kinetics of the interactions (primarily seen in the dissociation phase) were significantly influenced by single amino acid substitutions in the NNRTI binding pocket. MIV-170 was most resilient to these changes.

Due to the complex interaction and slow dissociation between HIV-1 RT and the NNRTIs [5], regression analysis using a 1:1 interaction model was unsatisfactory in describing the interactions and extracting the kinetic parameters (data not shown). More complex models were also unsatisfactory and unsuitable for the regression analysis of the limited data set that could be obtained for MIV-170, the compound of interest. Even if larger data sets, allowing for a regression analysis, in principle could be obtainable for some of the faster dissociating reference compounds and the mutants, it would not provide the information required to understand the characteristics of MIV-170. In addition, the absence of a regeneration procedure also prevented normalization of surfaces with a positive control and determination of the maximal signals for each compound, also of importance for quantification of parameters. The comparison of the interactions of NNRTIs with their target and the effect of the amino acid substitutions in the NNRTI binding pocket on the interaction was consequently restricted to a qualitative analysis of the sensorgrams.

\subsubsection{Qualitative analysis of sensorgrams}

In order to establish the kinetic mechanism of the interaction and qualitatively determine the difference in the kinetics for the different inhibitors, sensorgrams were simulated with a set of mechanistic models representing steps of the complex mechanism previously found to describe the interactions of NNRTIs with the K103N variant of HIV-1 RT 
[5]. An induced fit mechanism was the simplest model that captured the basic features of the experimental data (Fig. 3). In this model, the equilibrium for the initial encounter complex, is governed by the association and the dissociation rate constants $\left(k_{1}\right.$ and $k_{2}$, respectively) while the second equilibrium, representing the conformational change between the primary enzymeinhibitor complex $\left(\mathrm{E}_{1} \mathrm{I}\right)$ and the induced complex $\left(\mathrm{E}_{2} \mathrm{I}\right)$, is balanced by $k_{3}$ and $k_{4}($ Fig. 3$)$.

The simulation was performed with a single concentration of inhibitor $(5.12 \mu \mathrm{M}$, i.e. the one used in the current experiments) and a value for $k_{l}\left(1 \cdot 10^{4} \mathrm{M}^{-1} \mathrm{~s}^{-1}\right)$ similar to that previously determined for nevirapine and delavirdine and the K103N variant and a value for $k_{3}\left(5 \cdot 10^{-2} \mathrm{~s}^{-}\right.$ ${ }^{1}$ ) that was found to give sensorgrams similar to the experimental sensorgrams (this parameter was not quantified in the former study). The rate constant $k_{2}$ for the dissociation of the encounter complex was varied from 0.0001 to $1 \mathrm{~s}^{-1}$ (covering the range found in [5]), but with two different values for $k_{4}$, i.e. $1 \cdot 10^{-5} \mathrm{~s}^{-1}$ (Fig. 4A) or $1 \cdot 10^{-2} \mathrm{~s}^{-1}$ (Fig. 4B), representing "fast" and "slow" relaxation of the induced complex (relative both $k_{2}$ and $k_{3}$ ). A series of simulations with other parameter values, that support the selected values and show the effect of variations in the rates, is presented in Supplementary Material. Simulation of data using a selected fit model (i.e. with a pre-equilibrium between two forms of the free enzyme) was also tried, but did not give sensorgrams resembling the experimental sensorgrams, with any combinations of parameters tried (data not shown). A model including both the selected fit and induced fit steps was also used and resulted in sensorgrams very similar to the experimental data (Supplementary material, Figure SM3). However, as the aim was simply to identify the primary steps and the order of magnitude of the rate constants of the interaction it was not meaningful to extract any details from this simulation considering the large number of variables involved.

The simulation shows that the maximal signal level is predominantly influenced by $k_{2}$, i.e. the rate by which the primary enzyme-inhibitor complex dissociates into free enzyme and 
inhibitor (Fig. 4A). A change in the rate of relaxation $\left(k_{4}\right)$ from the induced complex into the primary enzyme-inhibitor complex does not affect the maximal signal level, but indeed the slope of the dissociation curve (Fig. 4B). In other words, a compound can reach high complex levels due to a low $k_{2}$, but show fast dissociation due to a high $k_{4}$, or only reach low complex levels despite little dissociation. Thus, the overall signal levels and the mere shape of the interaction curves revealed important pieces of information about the interaction mechanism and the different contributions of the dissociation and association rates to the overall affinity.

\subsection{2. $M I V-170$}

The sensorgrams showed that, of all tested compounds, MIV-170 blocks all variants of HIV-1 RT most efficiently over time. This is a result of the highest level of complex concentration formed (maximum signal level at the end of the injection) and the slowest apparent dissociation rate (Fig. 2). The compound appeared to interact essentially irreversibly since no dissociation was detected during the dissociation phase, measured for up to $10 \mathrm{~min}$ (not shown). The experimental sensorgrams corresponded to the simulation with very slow $k_{2}$ and $k_{4}$ values (exemplified in Fig. $4 \mathrm{~A}$ by $1 \cdot 10^{-4} \mathrm{~s}^{-1}$ and $1 \cdot 10^{-5} \mathrm{~s}^{-1}$, respectively).

\subsubsection{TMC-120}

TMC-120 was the only other compound showing essentially irreversible interactions with the targets. But this compound gave atypical sensorgrams with all four protein surfaces, with very slow apparent rates of association. This type of sensorgram is characteristic for interactions with compounds that are barely soluble under the experimental conditions (see discussion). Even the qualitative analysis therefore becomes difficult for this compound. But it can be concluded that, although the magnitude of $k_{2}$ cannot be estimated, the magnitude of $k_{4}$ must be very slow, i.e. similar to that for MIV-170. 


\subsubsection{Delavirdine, efavirenz and nevirapine}

Two of the NNRTIs in clinical use (delavirdine and efavirenz) also formed stable complexes with the wild type enzyme, but the complexes with the resistant variants were clearly weaker (Fig. 2). The amount of complex formed was also lower than for MIV-170. Delavirdine appeared to have a faster $k_{4}$ than the other compounds, especially with the substituted variants, as suggested by the simulation. Efavirenz appeared to have a faster $k_{2}$ than MIV-170 and delavirdine, explaining why it did not reach the same levels as the other compounds.

The third NNRTI in clinical use, nevirapine, interacted only weakly with the wild type, as indicated by the low signal levels, but the simulation indicated that this could be a result of a relatively fast $k_{2}$ rather than a slow $k_{1}$. Despite the low signal levels, it was possible to see that nevirapine, at least for wild type HIV RT, had a relatively slow $k_{4}$.

\subsection{Antiviral efficacy}

In order to determine how the interaction kinetics correlate with antiviral efficacy, the anti-HIV effect of the inhibitors was also analyzed. It was initially tested in a standard assay with MT-4 cells infected with wild type virus and three mutant viral strains with resistance to NNRTIs, as shown in Table 1. MIV-170 was the most potent inhibitor, with $\mathrm{nM} \mathrm{EC}_{50}$ values for all tested mutants. Nevirapine and delavirdine showed little effect, essentially only with the wild type virus. TMC-120 and efavirenz were effective with the wild type virus, and to varying degrees with the mutant virus strains.

The effect of the compounds against a wild type Ba-L virus (subtype B, R5) was also tested in PBMCs (Table 2) as these cells represent a real HIV-1 target cell type. The compounds were constantly present in the growth medium during the 14 days long experiments in order to mimic in vivo microbicide use, where an intra-vaginal ring secretes inhibitor constantly or a gel is applied on a regular basis. The assay duration was established 
to 14 days in order to detect possible breakthrough infections that were barely, or not at all, detectable at earlier time points. Again, analysis revealed that MIV-170 had the highest efficacy of all tested compounds.

\subsection{Correlation between interaction kinetic profile and antiviral efficacy}

A quantitative correlation analysis of the interaction data and the cell-based data was not possible since the kinetic rate constants for the interactions could not be determined. Nevertheless, the comparison between experimental and simulated sensorgrams revealed important kinetic characteristics of the inhibitors that could be used in a qualitative correlation analysis.

It was clear that the best antiviral efficacy was achieved when both $k_{2}$ and $k_{4}$ were very slow, as exemplified by MIV-170. The poor antiviral effect by delavirdine was attributed to a relatively fast $k_{4}$, while the poor antiviral efficacy of nevirapine was interpreted to be primarily an effect of a relatively fast $k_{2}$. Efavirenz differed from MIV-170 by having a faster $k_{2}$. Thus, the qualitative kinetic analysis allowed the explanation of critical kinetic features that correlated with antiviral efficacy.

\section{DISCUSSION}

One of the aims of the present study was to investigate if the novel NNRTI MIV-170 has kinetic properties suitable for use as a microbicide against HIV transmission. Since the long-term effect of an antiviral drug and a microbicide is expected to be dependent on the stability of the inhibitor-target complex, i.e. the rate of dissociation, we were particularly interested in studying the importance of slow dissociation for antiviral effect. This was evaluated in a physiological context by analyzing the correlation between the interaction kinetic parameters and antiviral activity in cells. 
The choice of biosensor-based interaction analysis for this study was based on our experience that it can provide detailed information on the interaction between NNRTIs and the enzyme [5-7], although not as mechanistically revealing as the pre-steady state kinetic analysis of the enzyme activity [14] previously used to determine the mechanism of NNRTI inhibition. However, there were several experimental challenges to overcome in the present study. One concerned the very tight complexes formed by most of the inhibitors with the enzyme, especially with the wild type enzyme form. In the previous studies, drug resistant enzyme variants and inhibitors that allowed regeneration of the sensor surfaces and repeated injections over the same surface were used. The sensorgrams could then be analyzed by regression analysis and some parameters quantified. In this study it was essential to perform experiments also with inhibitors and enzyme forms where regeneration was not possible, e.g. wild type. For these interactions the dissociation rate was too slow for the estimation of kinetic constants or affinities even if a simple 1:1 model had been an acceptable approximation [15]. Simulation of sensorgrams was therefore found to be very useful (see below).

The possibility of quantitatively analyzing interactions with very slow dissociation by use of simulation is expected to be very useful for lead-target interaction where ranking of compounds with respect to significant kinetic parameters is very difficult.

Previous studies have shown that the use of a higher temperature than $25^{\circ} \mathrm{C}$ for the experiments increases the dissociation rate [7]. However, we did not expect that a rise in temperature to e.g. physiologically relevant $37^{\circ} \mathrm{C}$, would have such an influence on the very slowly dissociating MIV-170 to resolve the regeneration problems experienced.

Another problem was the low solubility of at least one of the compounds, TMC-120. Poor solubility can result in a significantly lower effective free concentration of the analyte in the flow cell than the nominal concentration. Since the observed rate of association is directly 
correlated with the concentration of analyte, even small deviations in the concentration can be detected in the association phase. The observed rate of dissociation, on the other hand, is not directly influenced by the solubility of the analyte since it is only dependent on the dissociation rate constant $\left(k_{2}\right)$ and the concentration of the protein-ligand complex. However, if the concentration of analyte is very low, the system may not reach steady-state during the injection time. The amount of protein-ligand complex will then easily be underestimated in comparison to the amounts of complex formed with compounds that reach steady-state during the injection, thereby indirectly affecting the observed dissociation rate.

TMC-120 is known to have a very low solubility $(<0.3-1.2 \mu \mathrm{M})[16]$ and did not reach steady-state for any of the interactions studied. The compound showed an atypical behavior with all four enzymes, clearly indicating an inherent property independent of the interaction with the proteins, in accordance with a very low solubility. Although the TMC-120 sensorgrams do not accurately reflect the interaction mechanism, they clearly show that the compound dissociates very slowly (if at all). The other NNRTIs lacked the solubility problems of TMC-120, with MIV-170 being readily soluble up to $20 \mu \mathrm{M}$ (unpublished data). The possibility that solubility was a problem for other compounds was taken into consideration when analyzing the data, but in practice it only influenced the interpretation of the data for TMC-120.

The focus on kinetics in the present study was based on an expectation that the antiviral effect of NNRTIs is dependent on the kinetics of interactions rather than the equilibrium behavior, e.g. as described by $\mathrm{K}_{\mathrm{D}}, \mathrm{K}_{\mathrm{i}}$ or $\mathrm{IC}_{50}$ values. It has previously been proposed that a tight-binding mode of inhibition is essential for anti-HIV-1 virucidal activity of NNRTIs [17] and NNRTIs have been described as tight-binding (efavirenz) or rapid equilibrium (nevirapine and delavirdine). This terminology is, however, founded on an equilibrium-based analysis of reversible interactions rather than the time-resolved analysis that has been performed in the 
present study. As shown here, these terms are misleading for this type of inhibitors since an equilibrium-based approach is not appropriate for compounds that are truly "tight-binding", in the sense that they dissociate very slowly rather than simply having high affinities (low $\mathrm{K}_{\mathrm{D}^{-}}$ values) [18]. The use of enzyme inhibition data that does not account for irreversible inhibition mechanisms is therefore not relevant.

This study also illustrates the importance of determining the mechanisms of proteinligand interactions as they are often more complex than simple 1:1 interactions. HIV-1 RT has previously been shown to interact with NNRTIs in a complex mechanism involving 1) a rate limiting pre-equilibrium between two unbound forms of the enzyme (i.e. selected fit [19]), 2) a slow conformational change (i.e. induced fit [19]), as well as 3 ) a heterogeneity in the immobilized protein [5, 6]. However, the simulation showed that an induced fit mechanism alone could describe the dominating features of the current interactions. This may appear inconsistent seeing that the previous study concluded that the interaction was primarily selected fit, even if slow conformational changes also were inferred. But two datasets for the same target can be described primarily by different mechanisms if the mechanisms can be seen as parts of the same overall mechanistic model and that the dominant mechanism is defined by which steps are rate limiting [19]. The previous dataset, performed with the K103N variant and with inhibitors tested in concentration series, was best described by a selected fit mechanism for three out of four studied inhibitors, while the fourth compound (nevirapine) was adequately described by a simple 1:1 model [5]. Here we show that changes in the target (i.e. resistance mutations) may also influence the mechanism. Also the experimental conditions can have an impact as they may influence the relative rates of binding and conformational transitions in proteins.

Consequently, for NNRTI interactions with HIV-1 RT there are multiple equilibria that contribute to the "apparent dissociation rate". In the induced fit mechanism there is only one 
true dissociation rate constant $\left(k_{2}\right)$. This makes it clear that a good understanding of the interaction mechanism is essential before the critical kinetic features of a certain proteinligand interaction can be defined. Our interest in resolving the mechanism and the kinetics for NNRTIs was to correlate these features with the efficacy of the compounds in cell culture. Although it was frustrating that the rate constants could not be quantified, the simulations were very powerful in revealing the critical kinetic and mechanistic features for efficacy.

It was clear that the antiviral effect of the compounds was dependent on the rate of both the dissociation rate constant for the encounter complex $\left(k_{2}\right)$ and the relaxation from the induced complex $\left(k_{4}\right)$. The least effective compound in cell culture, nevirapine, was apparently compromised by a fast $k_{2}$ rate, preventing the efficient formation of enzymeinhibitor complex. The poor antiviral efficacy of delavirdine was more puzzling, as it clearly formed large amounts of complex which appeared to be stable. However, careful scrutiny of the sensorgrams showed that the apparent rate of dissociation was faster than for MIV-170. It can be speculated that small differences in rate constants (also inferred from the simulation in Fig. 4A) can have significant effects during the much longer experiment performed when measuring antiviral efficacy in a cell culture based assay. This is supported by comparing the effect of mutations on the interactions with the compounds. Interestingly, also the value of $k_{2}$ was important, and correlated with the antiviral effect of the compounds, with MIV-170 being the most effective compound in cell culture, and nevirapine the least effective. Consequently, slow apparent dissociation alone, represented by the inability to detect dissociation, was not enough to explain the antiviral efficacy of the compounds.

The simulations also clearly show how differences in rate constants influence the amount of complex formed. In the case of viral enzymes, it is critical to block any enzyme present. This is dependent on the kinetics as well as the inhibitor concentration. Of the studied inhibitors MIV-170 resulted in the largest amount of complex, and consequently allows the 
least amount of enzyme to be active (everything else being equal). We have already shown that the amount of blocked target is a critical parameter for HIV-1 protease, correlating better with antiviral efficacy than dissociation rate alone [20]. The focus on residence time of drugs can obviously be misleading for cases where residual enzyme activity can be problematic.

There are obvious limitations in using in vitro data to predict antiviral effects in vivo since in vivo data are influenced by more complex mechanistic features that cannot be reproduced in vitro. In addition, it is generally difficult to compare viral efficacy data with in vivo studies due to differences in viral strains and experimental conditions. A recent inhibition study with TMC-120 (dapivirine), delavirdine, efavirenz and nevirapine showed the same relative efficacy as in this study, qualitatively supporting the current results [10]. The correlation between biosensor kinetics and cell culture efficacy of NNRTIs are further supported by previous biosensor studies performed on etravirine (TMC-125). These show that etravirine, which is a potent inhibitor of viral replication in cell culture, dissociated slowly for the majority of the HIV-1 RT variants (9).

The compound of interest, MIV-170, did not dissociate with a measurable rate from any of the enzyme variants during the dissociation used in these experiments (10 min). Although it was not possible to detect and quantify the dissociation of MIV-170 it is not excluded that it does in fact dissociate, but it clearly does so very slowly. The only other compound with slow dissociation from all enzyme variants, TMC-120, gave considerably lower signal levels due to solubility problems. But despite these problems, the dissociation rate is interpreted to be realistic. Although delavirdine and efavirenz interact tightly with wild type HIV-1 RT, they are greatly affected by the substitutions in the binding site, as also reflected in the resistance towards these drugs by viruses with these mutations.

With respect to the use of NNRTIs as topical microbicides, it is essential to use a compound that can block the target essentially irreversibly over time. This should apply to 
both wild type and mutant strains. Although side-effects are expected to be less of a problem for microbicides as compared to a systemically delivered drug, it is still desirable to be able to use as low concentrations as possible. MIV-170 is clearly the most suitable compound of those tested here, at least from a kinetic perspective. In addition to the advantages of MIV-170 for use as a microbicide revealed by the present studies, other data suggests that systemic levels of the inhibitor will be small as a result of a rapid liver metabolism (unpublished observations). This will lower the risk of resistance development towards MIV-170 upon topical administration. A low protein binding and high chemical stability (unpublished observations) are further properties making MIV-170 suitable for a vaginal microbicide. Obviously other factors also need to be considered, but they were outside the scope of this study. Additional tests with HIV-1 subtype A and C and an evaluation of the ability of MIV170, in a vaginal formulation, to prevent Simian-Human immunodeficiency virus (SHIV) infection in monkeys, would be the next step.

\section{Conclusions}

This study illustrates that a biosensor-based method for studying the direct interaction between the NNRTI and its target can be used to identify compounds with suitable kinetic features for antiviral efficacy. Simulation of sensorgrams was useful for both establishing the interaction mechanism and identifying critical parameters for formation of large amounts of stable inhibitor-target complex. It is clear that the cell culture efficacy is an effect of the kinetics of both equilibria involved in an induced fit mechanism, emphasizing the importance of resolving the kinetic mechanism for drug-target interactions. It also reveals that long residence time (the time during which the target is blocked by the inhibitor) is not a good parameter for judging the efficacy of NNRTIs as the amount of complex is also a critical parameter. Finally, the results confirm that the kinetics, resulting in essentially irreversible 
binding of MIV-170 to wild type and NNRTI-resistant mutants of HIV RT, makes MIV-170 very interesting for development as a microbicide.

\section{Acknowledgements}

Thanks to Matthis Geitmann for critically reading the manuscript. This work was supported by SIDA, the Swedish International Development Cooperation Agency (Malin Elinder) and the Research foundation - Flanders (FWO) Belgium (PhD fellowship to Philippe Selhorst). The authors thank GE Healthcare Biosciences for the use of the Biacore A100 instrument for the interaction experiments.

\section{REFERENCES}

[1] Gray RH, Wawer MJ. Randomised trials of HIV prevention. Lancet 2007;370:200-1.

[2] Cohen J. Retrovirus meeting. Back-to-basics push as HIV prevention struggles. Science 2008;319:888.

[3] Sahlberg C, Zhou X-X. Development of Non-Nucleoside Reverse Transcriptase Inhibitors for Anti-HIV Therapy. Anti-Infective Agents in Medicinal Chemistry 2008;7:101-17.

[4] Nuttall JP, Thake DC, Lewis MG, Ferkany JW, Romano JW, Mitchnick MA. Concentrations of dapivirine in the rhesus macaque and rabbit following once daily intravaginal administration of a gel formulation of [14C]dapivirine for 7 days. Antimicrobial agents and chemotherapy 2008;52:909-14.

[5] Geitmann M, Unge T, Danielson UH. Biosensor-based kinetic characterization of the interaction between HIV-1 reverse transcriptase and non-nucleoside inhibitors. Journal of Medicinal Chemistry 2006;49:2367-74. 
[6] Geitmann M, Unge T, Danielson UH. Interaction kinetic characterization of HIV-1 reverse transcriptase non-nucleoside inhibitor resistance. Journal of Medicinal Chemistry 2006;49:2375-87.

[7] Geitmann M, Danielson UH. Additional level of information about complex interaction between non-nucleoside inhibitor and HIV-1 reverse transcriptase using biosensor-based thermodynamic analysis. Bioorganic \& Medicinal Chemistry 2007;15:7344-54.

[8] Geitmann M, Elinder M, de Esch I, Källblad P, Danielson UH. Fragment screening using SPR biosensors: Selection of hits for HIV-1 reverse transcriptase. Fragment Based Lead Discovery Conference. San Diego, 2008.

[9] Elinder M, Nordstrom H, Geitmann M, Hamalainen M, Vrang L, Oberg B, et al. Screening for NNRTIs with Slow Dissociation and High Affinity for a Panel of HIV-1 RT Variants. Journal of Biomolecular Screening 2009;14:395-403.

[10] Fletcher P, Harman S, Azijn H, Armanasco N, Manlow P, Perumal D, et al. Inhibition of human immunodeficiency virus type 1 infection by the candidate microbicide dapivirine, a nonnucleoside reverse transcriptase inhibitor. Antimicrobial agents and chemotherapy 2009;53:487-95.

[11] Bhikhabhai R, Carlsson T, Unge T, Lovgren S, Strandberg B. Increased yield of homogeneous HIV-1 reverse transcriptase (p66/p51) using a slow purification approach. J Chromatogr 1993;639:67-74.

[12] Hogberg M, Sahlberg C, Engelhardt P, Noreen R, Kangasmetsa J, Johansson NG, et al. Urea-PETT compounds as a new class of HIV-1 reverse transcriptase inhibitors. 3. Synthesis and further structure-activity relationship studies of PETT analogues. J Med Chem 1999;42:4150-60. 
[13] Beirnaert E, Willems B, Peeters M, Bouckaert A, Heyndrickx L, Zhong P, et al. Design and evaluation of an in-house HIV-1 (group M and O), SIVmnd and SIVcpz antigen capture assay. J Virol Methods 1998;73:65-70.

[14] Spence RA, Kati WM, Anderson KS, Johnson KA. Mechanism of inhibition of HIV-1 reverse transcriptase by nonnucleoside inhibitors. Science 1995;267:988-93.

[15] Önell A, Andersson K. Kinetic determinations of molecular interactions using Biacore--minimum data requirements for efficient experimental design. J Mol Recognit 2005; 18:307-17.

[16] Gupta J, Sharma P, Romano J, Garg S. Application of nanotechnology to enhance the solubility of poorly water soluble drug Dapivirine. ISPE Australasia Conference. Sydney, Australia, 2009.

[17] Motakis D, Parniak MA. A tight-binding mode of inhibition is essential for antihuman immunodeficiency virus type 1 virucidal activity of nonnucleoside reverse transcriptase inhibitors. Antimicrob Agents Chemother 2002;46:1851-6.

[18] Williams JW, Morrison JF. The kinetics of reversible tight-binding inhibition. Methods Enzymol 1979;63:437-67.

[19] Weikl TR, von Deuster C. Selected-fit versus induced-fit protein binding: kinetic differences and mutational analysis. Proteins 2009;75:104-10.

[20] Shuman CF, Vrang L, Danielson UH. Improved structure-activity relationship analysis of HIV-1 protease inhibitors using interaction kinetic data. Journal of Medicinal Chemistry 2004;47:5953-61.

[21] Fletcher P, Harman S, Azijn H, Armanasco N, Manlow P, Perumal D, et al. Inhibition of human immunodeficiency virus type 1 infection by the candidate microbicide dapivirine, a nonnucleoside reverse transcriptase inhibitor. Antimicrob Agents Chemother 2009;53:487-95. 
1

2

3

4

5

6

7

8

9

10

11

12

13

14

15

16

17

18

19

20

21

22

23

24

25

26

27

28

29

30

31

32

33

34

35

36

37

38

39

40

41

42

43

44

45

46

47

48

49

50

51

52

53

54

55

56

57

58

59

60

61

62

63

64

Page 23 of 34 


\section{FIGURE LEGENDS}

FIG. 1. Structure of MIV-170

FIG. 2. Interaction profiles for 5.12 $\mu \mathrm{M}$ MIV-170 (MIV, red), delavirdine (DEL, green), efavirenz (EFV, cyan), TMC 120 (TMC, violet) and nevirapine (NVP, blue) with four different variants of HIV-1 RT (WT, K103N, Y181C and L100I). Sensorgrams from the two replicate experiments are shown overlaid. They are reference subtracted, solvent corrected, molecular weight adjusted and normalized with respect to the immobilization levels. The grey horizontal dashed lines and the grey boxes are inserted to facilitate visual analysis of the data.

FIG. 3. The scheme for an induced fit interaction mechanism. $E_{1}$ represents the free enzyme in its low energy ground state, I the free inhibitor, $E_{1} I$ the enzyme-inhibitor complex in its high energy state and $\mathrm{E}_{2} \mathrm{I}$ the low energy induced complex. The rate constants are designated $k_{1}, k_{2}, k_{3}$ and $k_{4}$.

FIG.4. Simulated sensorgrams representing interactions according to an induced fit mechanism for varying magnitudes of $k_{2}$. All sensorgrams were simulated with $k_{l}=1 \cdot 10^{4} \mathrm{M}^{-1} \mathrm{~s}^{-}$ ${ }^{1}, k_{3}=5 \cdot 10^{-2} \mathrm{~s}^{-1}$ and the same concentration of enzyme $(5.12 \mu \mathrm{M})$. The dissociation rate constant $k_{2}$ was varied from $0.0001 \mathrm{~s}^{-1}$ (top) to $0.001,0.01,0.1$ and $1 \mathrm{~s}^{-1}$ (bottom). The values for $k_{4}$ were different in the two simulations, with A) $k_{4}=1 \cdot 10^{-5} \mathrm{~s}^{-1}$ and B) $k_{4}=1 \cdot 10^{-2} \mathrm{~s}^{-1}$. (Additional simulated sensorgrams with other combinations of rate constants are shown in Supplementary Materials.)

TABLE 1. Anti-HIV effect of NNRTIs against wt and mutant HIV-1 in MT-4 cells ${ }^{\mathrm{a}}$ The strain $\left(\mathrm{HIV}-1_{\mathrm{IIIb}}\right)$ is defined with respect to the reverse transcriptase sequence. 
TABLE 2. Anti-HIV effect of NNRTIs against wt HIV-1 in PBMCs

${ }^{\mathrm{a}}$ The calculation of the $\mathrm{EC}_{50}$ values and their $95 \%$ confidence intervals $(\mathrm{CI})$ was based on data of three independent experiments using non-linear regression analysis.

${ }^{\mathrm{b}}$ The virus strain is subtype B HIV-1 Ba-L. 


\section{FIGURES AND TABLES IN COLOR}

\section{Figure 1}<smiles>NS(=O)(=O)c1ccc(Oc2ccc(NC(=O)N[C@@H]3[C@H]4COc5c(F)ccc(F)c5[C@@H]43)cc2)cc1</smiles> 


\section{Figure 2}
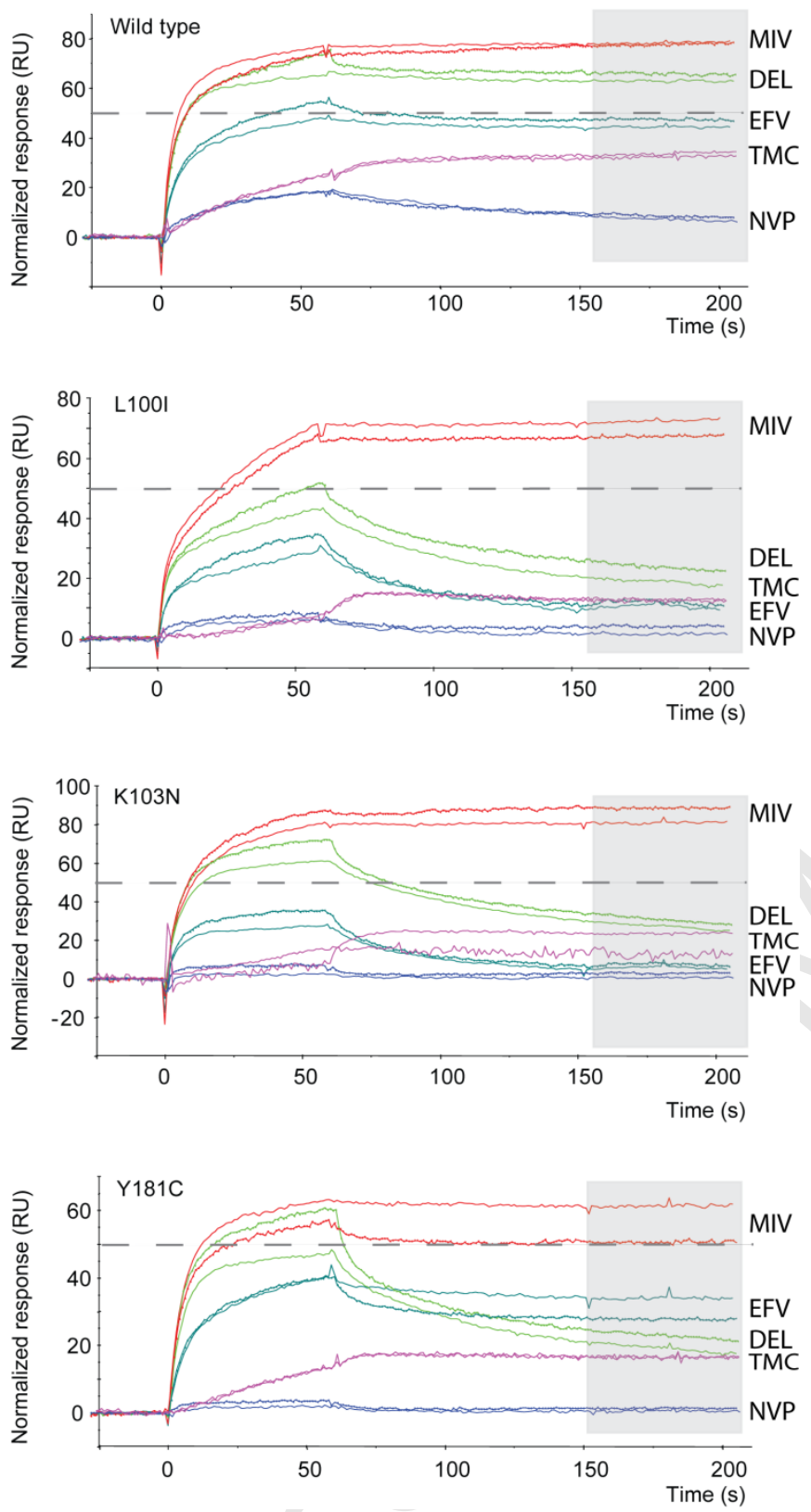
Figure 3

$$
\mathrm{E}_{1}+\mathrm{I} \underset{\mathrm{k}_{2}}{\stackrel{\mathrm{k}_{1}}{\rightleftarrows}} \mathrm{E}_{1} \mathrm{I} \underset{\mathrm{k}_{4}}{\stackrel{\mathrm{k}_{3}}{\rightleftarrows}} \mathrm{E}_{2} \mathrm{I}
$$




\section{Figure 4}

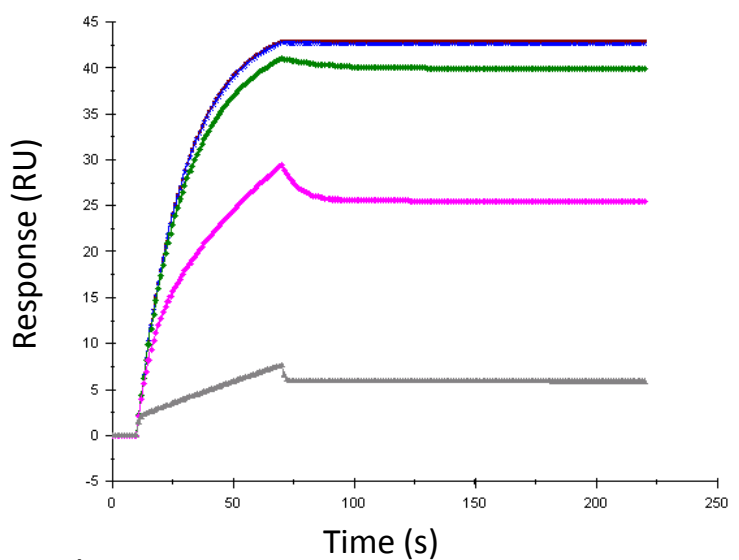

A)

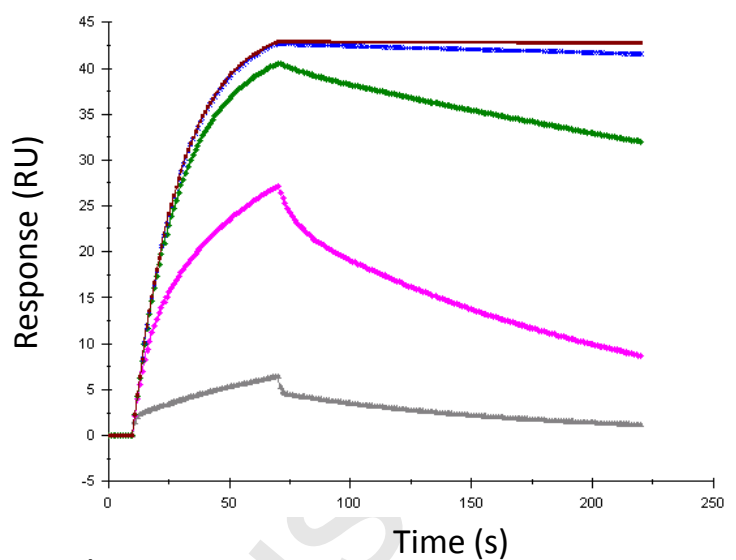

B) 
TABLE 1

\begin{tabular}{llllll} 
& \multicolumn{5}{c}{$\mathrm{EC}_{50}(\mathrm{nM})$} \\
\cline { 2 - 6 } Strain $^{\mathrm{a}}$ & MIV-170 & Delavirdine & Efavirenz & TMC-120 & Nevirapine \\
\hline Wt & 0.97 & 110 & 1.6 & 1.2 & 170 \\
L100I & 9 & 7900 & 88 & 40 & 1200 \\
K103N & 3.2 & 6400 & 20 & 5.5 & $>10000$ \\
Y181C & 5.3 & 5000 & 3.9 & 16 & $>10000$
\end{tabular}

TABLE 2

\begin{tabular}{|c|c|c|c|c|c|}
\hline \multirow[b]{2}{*}{ Strain $^{b}$} & \multicolumn{5}{|c|}{$\mathrm{EC}_{50}(\mathrm{nM})(95 \% \mathrm{CI})^{\mathrm{a}}$} \\
\hline & MIV-170 & Nevirapine & Delavirdine & Efavirenz & TMC-120 \\
\hline Wt HIV-1 & $2.1(0.2-25)$ & $370(244-55$ & $190(72-533)$ & 4.9 (3.1-7.' & $3.8(2.8-5$. \\
\hline
\end{tabular}




\section{FIGURES AND TABLES IN BLACK AND WHITE}

\section{Figure 1}<smiles>NS(=O)(=O)c1ccc(Oc2ccc(NC(=O)N[C@H]3[C@H]4COc5c(F)ccc(F)c5[C@@H]43)cc2)cc1</smiles> 


\section{Figure 2}
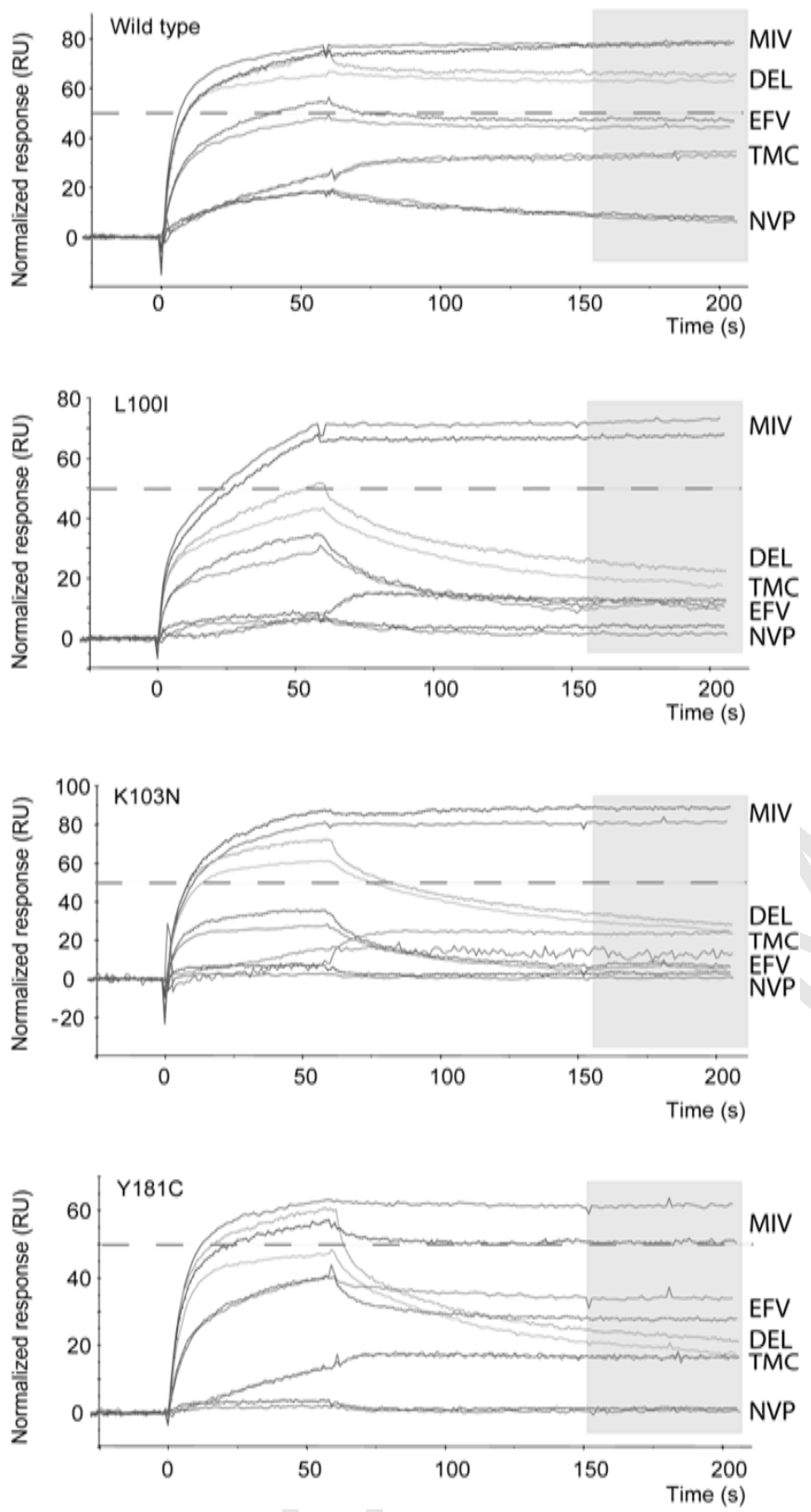

Figure 3

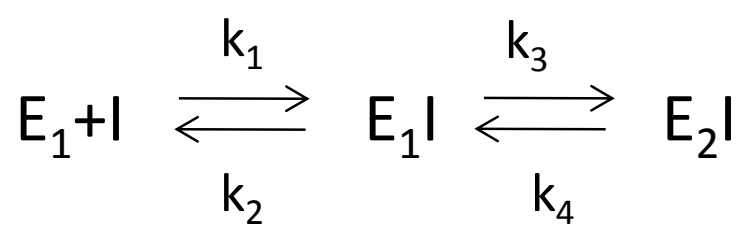




\section{Figure 4}

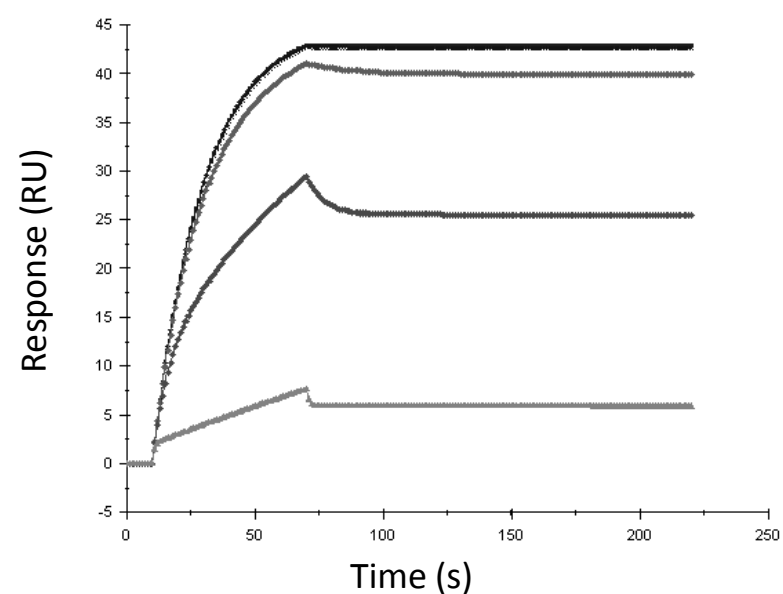

A)

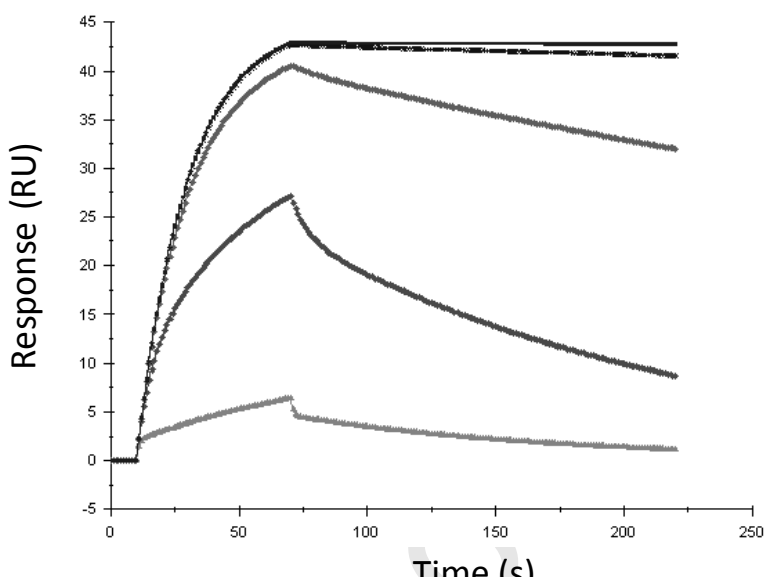

B)

$\mathrm{EC}_{50}(\mathrm{nM})$

\begin{tabular}{|c|c|c|c|c|c|}
\hline $\begin{array}{l}\text { Strain }^{\mathrm{a}} \\
(\text { HIV-1 } \\
\text { IIIb })\end{array}$ & MIV-170 & Delavirdine & Efavirenz & TMC-120 & Nevirapine \\
\hline $\mathrm{Wt}$ & 0.97 & 110 & 1.6 & 1.2 & 170 \\
\hline L100I & 9 & 7900 & 88 & 40 & 1200 \\
\hline K103N & 3.2 & 6400 & 20 & 5.5 & $>10000$ \\
\hline Y181C & 5.3 & 5000 & 3.9 & 16 & $>10000$ \\
\hline
\end{tabular}

TABLE 2

\begin{tabular}{|c|c|c|c|c|c|}
\hline Strain ${ }^{b}$ & MIV-170 & Nevirapine & Delavirdine & Efavirenz & TMC-120 \\
\hline Wt HIV-1 & $2.1(0.2-25)$ & $370(244-558)$ & $190(72-533)$ & $4.9(3.1-7.7)$ & $3.8(2.8-5.1)$ \\
\hline
\end{tabular}



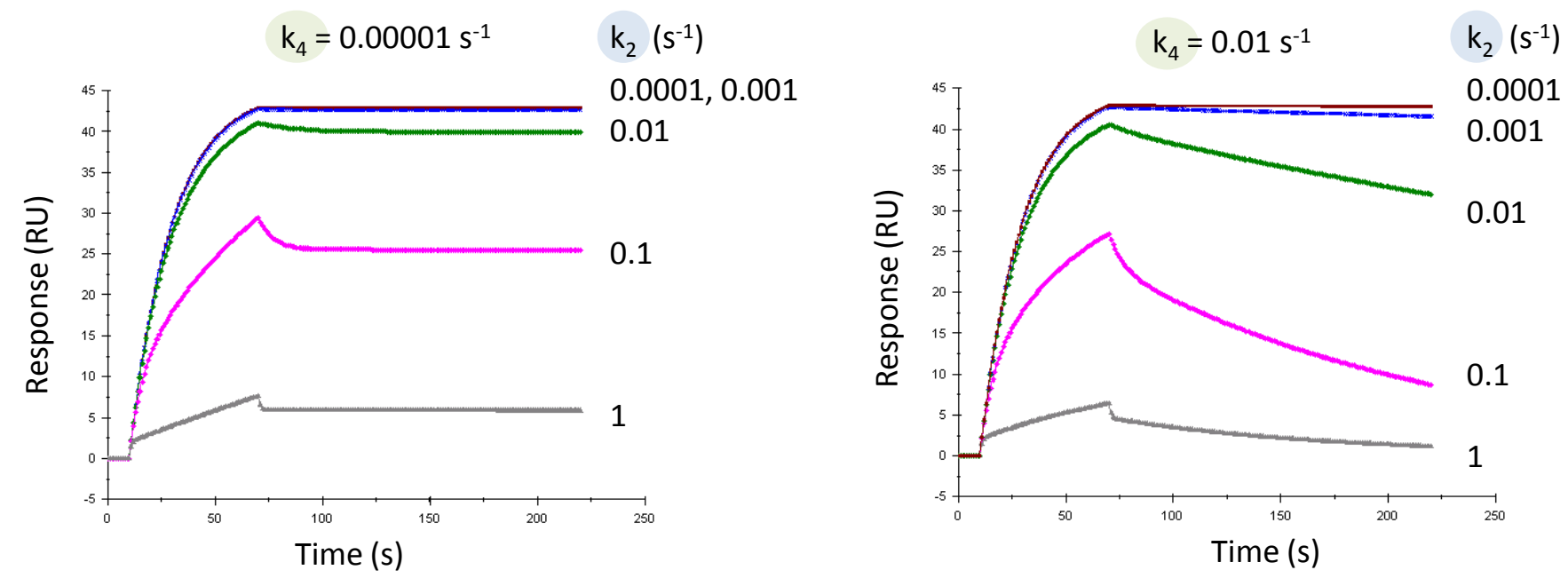

$$
E_{1}+1 \underset{k_{2}}{\stackrel{k_{1}}{\rightleftarrows}} E_{1}\left|\underset{k_{4}}{\stackrel{k_{3}}{\rightleftarrows}} E_{2}\right|
$$

Simulated sensorgrams representing an induced-fit mechanism with very slow dissociation rate $\left(k_{2}\right)$ and relaxation rate $\left(k_{4}\right)$ constants correspond to the experimental data and explain the high antiviral efficacy of the NNRTI MIV-170. 